\title{
A Theorem on Convex Hulls
}

\author{
W. A. Horn \\ Institute for Basic Standards, National Bureau of Standards, Washington, D.C. 20234
}

(July 1, 1969)

\begin{abstract}
Let $p$ be a point in the interior $K^{\circ}$ of the convex hull $K=K(S)$ of a bounded point-set $S$ in a real Hilbert space. A quantity $R(p)$ is determined such that every closed ball of radius $>R(p)$, if it contains $p$, must also meet $S$.
\end{abstract}

Key words: Convex geometry; convex sets.

\section{Introduction}

Let $K=K(S)$ be the convex hull of a bounded point-set $S$ in a real Hilbert space $E$. This note is concerned with the following questions, which arise for example ${ }^{1}$ in connection with the theory of " $\epsilon$-convex sets."

Consider a point $p \epsilon K$. Is it true that every large enough closed ball, which contains $p$, must also meet $S$ ? How large is "large enough"?

It is readily seen that the answer to the first question is in general negative if $p$ lies in the boundary $\partial K$ of $K$ (unless, of course, $p \epsilon S$ ). Thus it will be assumed throughout that $p$ lies in the interior $K^{\circ}$ of $K=K(S)$.

With the notation

$$
\begin{aligned}
& \delta=\operatorname{diam}(S)=\operatorname{diam}(K)<\infty, \\
& \Delta=d(p, \partial K) \leqslant \delta,
\end{aligned}
$$

define $R^{*}(p)$ and $R(p)$ by

$$
\begin{aligned}
R^{*}(p) & =\delta^{2} / 2 \Delta, \\
R(p) & =R^{*}(p)+\delta .
\end{aligned}
$$

For $p \epsilon K^{\circ}$, with $K, S, E, R^{*}(p)$ and $R(p)$ as above, the following results will be proved:

Theorem 1: Every closed ball $\mathrm{B}$ with radius $>\mathrm{R}^{*}(\mathrm{p})$ for which $\mathrm{p} \epsilon \mathrm{B}$ meets $\mathrm{S}$.

THEOREM 2: Every closed ball $\mathrm{B}$ with radius $>\mathrm{R}(\mathrm{p})$, which contains $\mathrm{p}$, meets $\mathrm{S}$.

\section{Proof of Theorem 1}

Let $B$ be a closed ball of radius $r>R^{*}(p)$, such that $p \epsilon \partial B$. We wish to show that $B$ meets $S$.

Let $c$ be the center of $B$, and $u$ the unit vector in the direction of $p-c$. Thus $p=c+r u$. Let $H_{1}$ be the closed hyperplane tangent to $B$ at $p$, i.e.

$$
H_{1}=p+\{y:(y, u)=0\} .
$$


Let $E_{1}$ be that one of the closed halfspaces of $E$ determined by $H_{1}$ which contains $c$ and thus contains $B$.

Since $r>R^{*}(p)=\delta^{2} / 2 \Delta$, we can choose $\eta<\Delta$ such that $r \eta>\delta^{2} / 2$. Let $H_{2}$ be the hyperplane parallel to $H_{1}$ which passes through the point $q=c+(r-\eta) u$. Then $H_{2}$ is at distance $\eta$ from $H_{1}$, and lies in $E_{1}$.

Let $E_{2}$ be that one of the closed halfspaces of $E$ determined by $H_{2}$ which does not contain $H_{1}$. Then $E_{2}-H_{2}$ is met by the open ball of radius $\Delta$ centered at p, e.g. at the midpoint of $q$ and $c+(r-\Delta) u$. This ball lies wholly in $K$, so $E_{2}-H_{2}$ meets $K$. Thus the closed convex set $E-\left(E_{2}-H_{2}\right)$ does not contain all of the convex hull $K=K(S)$, and so does not contain all of $S$. Hence $E_{2} \cap S$ is nonempty; to complete the proof of theorem 1, it suffices to prove that $E_{2} \cap S \subset B \cap S$.

Consider then any $x \in E_{2} \cap S$. We need to show that

$$
d(x, c)=\|x-c\| \leqslant r .
$$

Note that $x-c$ admits a unique orthogonal decomposition relative to $u$, i.e.,

$$
x-c=y+\theta u, \quad(y, u)=0 .
$$

This yields

$$
\|x-c\|^{2}=\|y\|^{2}+\theta^{2}
$$

Because $x \in E_{2}$, we have

$$
\theta \leqslant r-\eta
$$

Because $p \epsilon K$ and $x \epsilon S \subset K$, we have

$$
\begin{aligned}
\delta^{2} & =[\operatorname{diam}(K)]^{2} \geqslant\|x-p\|^{2} \\
& =\|(x-c)-(p-c)\|^{2}=\|y-(r-\theta) u\|^{2} \\
& =\|y\|^{2}+(r-\theta)^{2}=\|y\|^{2}+\theta^{2}+r^{2}-2 r \theta .
\end{aligned}
$$

Combining this with $(2.3)$ gives

$$
\|x-c\|^{2} \leqslant \delta^{2}+2 r \theta-r^{2}
$$

and application of (2.4) then yields

$$
\|x-c\|^{2} \leqslant \delta^{2}+2 r(r-\eta)-r^{2}=r^{2}-\left(2 r \eta-\delta^{2}\right) .
$$

Since $\eta$ was so chosen that $2 r \eta>\delta^{2},(2.1)$ is proved.

\section{Proof of Theorem 2}

We shall show that theorem 2 follows from theorem 1. Let $B$ be a closed ball of radius $r-R(p)$ such that $p \in B$. There are two cases to be considered, depending on the distance of $p$ from the center $c$ of $B$. Since

If $d(c, p) \leqslant R^{*}(p)=R(p)-\delta$, then $B$ contains the closed ball $B^{\prime}$ of radius $\delta$ centered at $p$.

$$
\delta=\operatorname{diam}(S)=\operatorname{diam}(K),
$$

while $p \epsilon K$, it follows that $K \subset B^{\prime} \subset B$ and thus that $S \subset B$. So $B$ certainly meets $S$.

Now suppose $d(c, p)>R^{*}(p)$. Let $B^{*}$ be the closed ball of radius $d(c, p)$ centered at $c$. Then $B^{*}$ has radius $>R^{*}(p)$, and $p \epsilon \partial B^{*}$. By theorem $1, B^{*}$ meets $S$. Since $B^{*} \subset B, B$ meets $S$. 